\title{
Distinct clinical and neuropathological features of G51D SNCA mutation cases compared with SNCA duplication and H50Q mutation
}

Aoife P. Kiely ${ }^{1}$ (D) Helen Ling ${ }^{1}$, Yasmine T. Asi ${ }^{1}$, Eleanna Kara ${ }^{9}$, Christos Proukakis ${ }^{5}$, Anthony H. Schapira ${ }^{5}$, Huw R. Morris ${ }^{5}$, Helen C. Roberts ${ }^{6}$, Steven Lubbe ${ }^{5}$, Patricia Limousin ${ }^{3}$, Patrick A. Lewis ${ }^{2,4}$, Andrew J. Lees ${ }^{1,2}$, Niall Quinn ${ }^{7}$, John Hardy ${ }^{1,2}$, Seth Love ${ }^{8}$, Tamas Revesz ${ }^{1}$, Henry Houlden ${ }^{2}$ and Janice L. Holton ${ }^{1 *}$

\begin{abstract}
Background: We and others have described the neurodegenerative disorder caused by G51D SNCA mutation which shares characteristics of Parkinson's disease (PD) and multiple system atrophy (MSA). The objective of this investigation was to extend the description of the clinical and neuropathological hallmarks of G51D mutant SNCA-associated disease by the study of two additional cases from a further G51D SNCA kindred and to compare the features of this group with a SNCA duplication case and a H50Q SNCA mutation case.

Results: All three G51D patients were clinically characterised by parkinsonism, dementia, visual hallucinations, autonomic dysfunction and pyramidal signs with variable age at disease onset and levodopa response. The H50Q SNCA mutation case had a clinical picture that mimicked late-onset idiopathic PD with a good and sustained levodopa response. The SNCA duplication case presented with a clinical phenotype of frontotemporal dementia with marked behavioural changes, pyramidal signs, postural hypotension and transiently levodopa responsive parkinsonism. Detailed post-mortem neuropathological analysis was performed in all cases. All three G51D cases had abundant a-synuclein pathology with characteristics of both PD and MSA. These included widespread cortical and subcortical neuronal a-synuclein inclusions together with small numbers of inclusions resembling glial cytoplasmic inclusions (GCls) in oligodendrocytes. In contrast the H50Q and SNCA duplication cases, had a-synuclein pathology resembling idiopathic PD without GCls. Phosphorylated a-synuclein was present in all inclusions types in G51D cases but was more restricted in SNCA duplication and H50Q mutation. Inclusions were also immunoreactive for the 5G4 antibody indicating their highly aggregated and likely fibrillar state.

Conclusions: Our characterisation of the clinical and neuropathological features of the present small series of G51D SNCA mutation cases should aid the recognition of this clinico-pathological entity. The neuropathological features of these cases consistently share characteristics of PD and MSA and are distinct from PD patients carrying the H50Q or SNCA duplication.
\end{abstract}

Keywords: Parkinson's disease, Multiple system atrophy, a-synuclein, Clinical features, Phosphorylation, Mutation, SNCA

\footnotetext{
* Correspondence: janice.holton@ucl.ac.uk

${ }^{1}$ Department of Molecular Neuroscience, Queen Square Brain Bank, UCL

Institute of Neurology, Queen Square, WC1N 3BG London, UK

Full list of author information is available at the end of the article
} 


\section{Background}

The SNCA gene encodes the $\alpha$-synuclein protein, which has a predicted molecular weight of $17 \mathrm{kDa}$, is expressed abundantly in human brain and is believed to function in vesicle recycling [1]. $\alpha$-Synucleinopathies, such as Parkinson's disease (PD), multiple system atrophy (MSA) and dementia with Lewy bodies (DLB), share the pathological hallmark of intracellular inclusions in which $\alpha-$ synuclein is a major constituent. In PD and DLB the pathological $\alpha$-synuclein inclusions are largely neuronal in the form of Lewy bodies (LB) and Lewy neurites (LN) while in MSA the most frequent site of aggregated $\alpha$-synuclein is the oligodendrocyte forming glial cytoplasmic inclusions (GCIs). Previously, multiplications (duplications and triplications) as well as several missense point mutations of the SNCA gene: A53T [2], E46K [3], A30P [4] and H50Q [5], were found to cause autosomal dominant PD. We recently reported a novel G51D SNCA mutation [6], which resulted in clinical and neuropathological features with some similarities to both PD and MSA. Affected family members developed early-onset Parkinson's disease with dementia. Neuropathological features included CA2-CA3 hippocampal and cortical neuronal loss with widespread, numerous neuronal $\alpha$-synuclein positive cytoplasmic inclusions together with smaller numbers of oligodendroglial inclusions that resembled the GCIs of MSA and so were referred to as GCI-like inclusions. A similar combined PD and MSA profile was also recently reported in a Finnish family carrying a novel A53E mutation [7]. Known SNCA mutations cluster in a putative protein loop, disruption of which may significantly alter the behaviour of the $\alpha$-synuclein protein by affecting lipid binding and fibril formation [8]. The neuropathological appearances associated with different mutations have varied considerably, but it is still unclear how alteration in $\alpha$-synuclein structure determines the neuropathology. A genetic cause has not been demonstrated in MSA, although recessive $C O Q 2$ mutations have been suggested to underlie a subset of familial MSA cases in the Japanese population [9].

Following our initial G51D mutation study, additional cases were reported in France [10] and Japan [11]. Affected family members in both reports had a similar clinical progression to that in our reported family, with early-onset levodopa-responsive parkinsonism accompanied by dementia. The clinical symptoms of the G51D mutation case described by Tokutake and colleagues closely resembled those in the proband of the family which we have reported [11]. Their case presented with levodopa-responsive parkinsonism at a young age (28 years), dementia, hallucinations and autonomic dysfunction. Lesage et al. noted widespread neuronal $\alpha$ synuclein pathology, which was similar in distribution and morphology to that which we observed: however, they did not report GCI-like inclusions [10].

We have recently identified a second British family with the G51D $\alpha$-synuclein mutation and have investigated post-mortem brain tissue from two affected family members. In the current study we sought to assess the spectrum and variability of clinical and neuropathological features in the three G51D cases (these two and our original case), and to determine whether there are particular phenotypic features that may suggest a G51D mutation and indicate that analysis of $S N C A$ is required.

For comparison, we analysed the clinical and neuropathological features in a H50Q mutation case and in a SNCA duplication case: some details of each of these cases have been published previously $[5,12]$.

We consider both the H50Q mutation case and the SNCA duplication case to be pertinent comparisons with the G51D cases. The H50Q mutation site is immediately adjacent to the G51D site within the region of $\alpha$ synuclein which is important for fibril formation and lipid binding [8]. It might therefore be expected that there would be strong similarities between the phenotypical profiles of this case and the G51D mutation cases. We previously reported extensive $\alpha$-synuclein pathology on post-mortem examination of a case with a large 6.4 Mb duplication of the SNCA locus [12]. This case was used as a further comparator as this previous report suggested that the duplication of SNCA can result in severe $\alpha$-synuclein pathology resembling that of G51D mutation [6, 12].

We have demonstrated in this detailed clinical and neuropathological study that G51D mutation cases share a constellation of features of parkinsonism with dementia, visual hallucination and autonomic dysfunction, with abundant $\alpha$-synuclein pathology with characteristics of both PD and MSA. The G51D mutation cases differ from the late-onset, relatively benign, PDlike presentation in the case with a H50Q mutation but share similarities with the SNCA duplication case. However, the neuropathological features of the G51D cases were distinct from those of H50Q mutation and SNCA duplication, although there were some common findings when the post-translational modifications of $\alpha$ synuclein were explored.

\section{Results}

The clinical features of all cases are summarised in Table 1.

\section{G51D case one: family one, patient II: 1 Fig. 1a}

We have previously described the clinical history of case one [6]. The index case was a British Caucasian male who presented at age 19 with left hand tremor and slowly progressive, asymmetrical, levodopa-responsive 
Table 1 Summary of clinical findings

\begin{tabular}{|c|c|c|c|c|c|}
\hline Case & Case one (G51D) & Case two (G51D) & Case three (G51D) & $\mathrm{H} 50 \mathrm{Q}$ & Duplication \\
\hline Age of onset (years) & 19 & 69 & 46 & 71 & 38 \\
\hline Disease duration (years) & 29 & 6 & 6 & 12 & 12 \\
\hline Presenting symptoms & $\begin{array}{l}\text { Resting hand } \\
\text { tremor, anxiety }\end{array}$ & $\begin{array}{l}\text { Resting hand tremor, anxiety } \\
\text { and depression }\end{array}$ & Resting hand tremor, depression & Resting hand tremor & Resting hand tremor and tongue tremor \\
\hline Final clinical diagnosis & $\begin{array}{l}\text { Familial pallidopyramidal } \\
\text { syndrome }\end{array}$ & $\begin{array}{l}\text { Parkinson's disease } \\
\text { with dementia }\end{array}$ & Parkinson's disease with dementia & Parkinson's disease & FTDP-17 \\
\hline Levodopa responsive & Good and sustained & Transient & Transient & Good and sustained & Transient \\
\hline Motor fluctuation & Yes & No & No & No & No \\
\hline Dystonia & $\begin{array}{l}\text { Wearing-off foot } \\
\text { dystonia }\end{array}$ & $\begin{array}{l}\text { Blepharospasm (unrelated to } \\
\text { dopamine replacement therapy) }\end{array}$ & No & $\begin{array}{l}\text { Blepharospasm } \\
\text { (unrelated to dopamine } \\
\text { replacement therapy) }\end{array}$ & $\begin{array}{l}\text { Blepharospasm and cervical dystonia } \\
\text { (unrelated to dopamine replacement } \\
\text { therapy) }\end{array}$ \\
\hline $\begin{array}{l}\text { Latency from first symptom of } \\
\text { onset of cognitive impairment } \\
\text { (years) }\end{array}$ & 8 & 2 & 2 & Not applicable & 9 \\
\hline Prominent cognitive impairment & Yes & Yes & Yes & No & Yes \\
\hline $\begin{array}{l}\text { Predominant frontal cognitive } \\
\text { impairment }\end{array}$ & No & $\begin{array}{l}\text { Yes (emotional lability, apathy, } \\
\text { disinhibition) }\end{array}$ & $\begin{array}{l}\text { Yes (perseveration, frontal executive } \\
\text { impairment, grasp reflex) }\end{array}$ & No & $\begin{array}{l}\text { Yes (obsessive behaviour, self-neglect, } \\
\text { markedly increased appetite, grasp reflex, } \\
\text { perseveration, motor recklessness) }\end{array}$ \\
\hline $\begin{array}{l}\text { Visual hallucinations } \\
\text { (unrelated to drug effect) }\end{array}$ & Yes & Yes & Yes & No & Yes \\
\hline Autonomic dysfunction & Yes & Yes & Yes & No & Yes \\
\hline $\begin{array}{l}\text { Pyramidal signs (pathological } \\
\text { reflexes and extensor plantar } \\
\text { response) }\end{array}$ & Yes & Yes & Yes & No & Yes \\
\hline Additional features & Myoclonus seizures & $\begin{array}{l}\text { Vertical supranuclear gaze palsy, } \\
\text { apraxia of eyelid opening }\end{array}$ & Not applicable & No & Not applicable \\
\hline Family history of parkinsonism & Father, sister & Mother, Aunt, brother, Son & $\begin{array}{l}\text { Mother, uncle, grandmother, } \\
\text { great aunt }\end{array}$ & No & $\begin{array}{l}\text { Father, male cousin, grandmother, two } \\
\text { great aunts }\end{array}$ \\
\hline
\end{tabular}


A
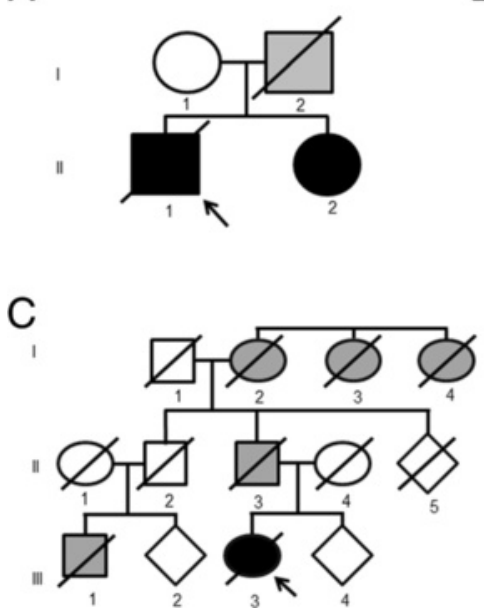
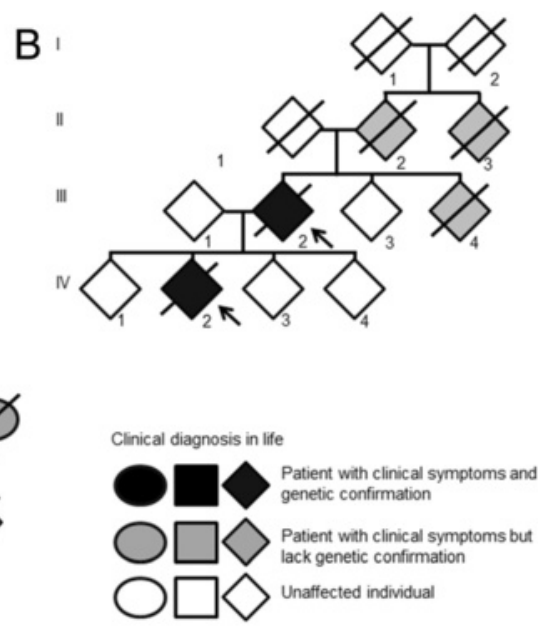

Fig. 1 Genetic Pedigrees. Simplified pedigree structures in which arrows are used to indicate the proband, circles indicate females, squares indicate males and diamonds indicate individuals of indeterminate or undisclosed gender, a G51D case one (patient II,1). The father of case one was diagnosed with PD (grey), his mother was unaffected (white). His sister carries the G51D mutation and developed PD symptoms at 40 years of age. $\mathbf{b}$ G51D cases two (patient III, 2) and three (patient IV, 2), Case two is the parent of case three. A sibling and two members of the previous generation of case two were diagnosed with PD without dementia (grey). c SNCA duplication case (patient III, 3), The father of the duplication case, paternal grandmother and two paternal great-aunts suffered from PD without documented dementia. Her paternal cousin was diagnosed with possible FTDP-17. The H50Q case did not have a family history of Parkinsonism

parkinsonism with marked motor fluctuation. Cognitive decline and visual hallucination started 9 years after disease onset. Autonomic dysfunction, pyramidal signs, myoclonus and seizures were also noted. The disease duration was 29 years. His father who had a background of depression and obsessional personality, also developed parkinsonism at age 39 followed by dementia, and died of sepsis at age 47 .

The index case's sister (patient II:2), who is alive and is now 49 years old, presented with resting tremor of the left hand at age 40 . In the first 5 years, her parkinsonism was slowly progressive but was well controlled by levodopa therapy, with occasional reports of end-of-dose wearing-off and dyskinesia. At age 47, she developed urinary urgency, incontinence and postural hypotension. She also started to fall and became confused with cognitive decline and visual hallucinations. She is now severely debilitated by memory impairment, disorientation, marked akinetic rigidity and urinary incontinence.

\section{G51D case two: family two, patient III: 2 Fig. $1 \mathrm{~b}$}

At age 69, this British Caucasian presented with resting tremor of the right hand, anxiety and depression. Examination revealed hypomimia, micrographia, bilateral resting hand tremor, asymmetrical bradykinesia and rigidity with parkinsonian gait and reduced arm swing. Hyperreflexia and mute plantar responses were noted. Initial examination confirmed a normal range of eye movements and normal saccades. Postural hypotension with orthostatic dizziness was documented and urinary urgency developed two years later. There was a good initial levodopa response, which waned after 3 years. At age 71 vivid dreams followed by bizarre behaviour, were reported by the spouse. Confusion and disorientation were frequent and disturbing, persistent visual hallucinations and paranoid delusions were problematic despite reducing the dosage of Ropinirole. A vertical supranuclear gaze palsy with very restricted upgaze and blepharospasm was noted. Rivastigmine was started which led to some improvements in clarity of thought, and fewer visual hallucinations. At age 73, the patient was withdrawn, emotionally labile and had minimal speech output. Episodes of confusion and wandering at night continued, the patient was dependent for all care and was incontinent of urine. In the last 2 years of life, severe dysphagia developed, there was no speech output, spasticity was marked, recurrent chest infections occurred, the patient was confined to bed and died at age 75 after a disease duration of 6 years.

A sibling (patient III: 4) also developed parkinsonism followed by dementia in the fourth decade of life. Clinical deterioration was rapid and death was reported within several years. Medical records and brain tissue were not available for examination.

\section{G51D case three: family two, patient IV: 2 Fig. 1 b}

At age 46, this British Caucasian, who was the child of case two, presented with several months history of resting tremor of the right hand and depressed mood. A diagnosis of Parkinson's disease was made and response to levodopa was good. At age 48, visual hallucinations 
developed, which did not improve despite withdrawal of dopamine agonist and monoamine oxidase B inhibitor. In the following year, word-finding difficulties were experienced with evidence of perseveration and frontal executive impairment. Motor symptoms and mobility began to deteriorate significantly and falls became a feature. By the age of 50 cognition was profoundly affected with markedly reduced speech production. Examination revealed hypomimia, normal saccadic and pursuit eye movements, bilateral resting hand tremor, bradykinesia, cogwheel rigidity and difficulty copying interlocking pentagons. There were frontal release signs including marked bilateral grasp reflex. Neuropsychometric assessment confirmed frontal and subcortical cognitive impairment. Single-photon emission computerised tomography (SPECT) scan was abnormal with signal reduction in the posterior cortical regions which was compatible with the clinical manifestation of cognitive impairment. At age 51, there was prominent behavioural disturbance, anxiety, irritability and persistent visual hallucinations. The patient become non-communicative, severely dysphagic and was incontinent of urine. Nursing home care was required and death occurred the following year aged 52 after a disease duration of 6 years.

\section{H50Q mutation case}

As previously reported [5], this British Caucasian female developed right hand tremor at age 71 with sustained benefit from levodopa therapy. By age 80 she had marked parkinsonism. She complained of mild memory impairment. Examination revealed tongue tremor, moderate bilateral resting hand tremor, bradykinesia, cogwheel rigidity, hyperreflexia and postural instability. At age 82, there was one report of confusion and hallucination which resolved after withdrawal of bromocriptine. There was no prominent motor fluctuation, cognitive impairment, behavioural change or autonomic dysfunction. She died at age 83 after a disease duration of 12 years. There was no family history of any neurological disorder.

\section{SNCA duplication case: patient III: 3 Fig. 1c}

The details of this case were previously published [12]. This British Caucasian female had longstanding extreme anxiety, panic disorders, hallucinations and a history of seizures. At age 38, she developed tremor of the right hand and the tongue, cervical dystonia, blepharospasm and falls. There was good initial levodopa response, lasting 8 years. At age 47, the most prominent features were obsessional behaviour, poor self-care and a profound increase in appetite, particularly for sweet food. She was diagnosed clinically as having possible frontotemporal dementia with parkinsonism-17 (FTDP-17) but subsequent genetic analysis did not reveal any $M A P T$ mutation. Mini mental state examination (MMSE) score was $24 / 28$ excluding a writing task, with most points being lost on attention. Examination showed normal eye movements, hypomimia, jaw tremor, asymmetrical resting tremor, bradykinesia, rigidity, parkinsonian gait with reduced arm swing, hyperreflexia and extensor plantar responses. Prominent frontal impairment was evident with bilateral grasp reflex, magnetic behaviour, perseveration on clapping task and motor recklessness. She also developed postural hypotension and autonomic function testing confirmed cardiovascular dysautonomia. Neuropsychometric findings were compatible with frontal and temporal impairments. She became bedbound and died at age 49 after a disease duration of 12 years. Her father, paternal cousin, paternal grandmother and two paternal great aunts all had a clinical diagnosis of PD, dementia or FTDP-17.

\section{Neuropathological data}

The neuropathological features of all cases are summarised in Table 3.

In our previous paper we analysed the neuropathology of G51D case one. Here we have compared case one with two further G51D cases from an independent kindred to assess the consistency of the neuropathological features associated with this mutation. The semiquantitative assessment of regional neuronal loss and both neuronal and glial $\alpha$-synuclein pathology is presented in Table 2, providing the range of pathological change seen in the three cases. All three G51D mutation cases shared the neuropathological hallmarks which defined case one. There was widespread neuronal and neuritic $\alpha$-synuclein pathology in all three cases: this included involvement of the neocortex, in addition to the striatum, limbic and brainstem regions. Representative images of these findings are shown in the CA3, caudate $(\mathrm{Cd})$, substantia nigra $(\mathrm{SN})$, putamen $(\mathrm{Pt})$ and dentate fascia (DF) in Fig. 2a. We previously described the varying morphology of the neuronal $\alpha$-synuclein cytoplasmic inclusions (annular, crescentic, globular, diffuse and neurofibrillary tangle-like). All three cases displayed the same spectrum of inclusion types and in similar distribution, although with some variation in severity. In all cases sparse GCI-like oligodendroglial inclusions were present in the white matter. These were most readily identified in the subcortical white matter, pontine base and cerebellar hemispheric white matter in all cases (Fig. 5a). In addition there were very occasional $\alpha$ synuclein positive coiled body-like glial inclusions as seen in cases of PD [13]. Some case-to-case variation was observed. In all three cases, the hippocampus showed marked $\alpha$-synuclein pathology, although the degree of neuronal loss in the CA2-CA3 region was variable between the cases, being most severe in cases one and two. The neocortex showed a similar degree of 
Table 2 Summary of neuropathological findings in three cases of G51D mutation

\begin{tabular}{|c|c|c|c|c|c|c|c|}
\hline & \multirow[t]{2}{*}{ Neuronal loss } & \multicolumn{5}{|l|}{$\begin{array}{l}\text { Neuronal a-synuclein } \\
\text { pathology }\end{array}$} & \multirow[t]{2}{*}{$\begin{array}{l}\text { Oligodendroglial } \\
\text { a-synuclein inclusions }\end{array}$} \\
\hline & & Annular or crescent & Globular & Diffuse & NFT-like & Threads & \\
\hline \multicolumn{8}{|l|}{ Cortex } \\
\hline Frontal & + & ++ & $+/++$ & + & $-/+$ & ++ & $-/+$ \\
\hline Motor & $-/+$ & ++ & $+/++$ & + & $-/+$ & $++/+++$ & $-/+$ \\
\hline Temporal & $+/+++$ & +++ & $+/++$ & $+/++$ & $-/+$ & +++ & $-/+$ \\
\hline Parietal & + & $+/+++$ & ++ & + & $-/+$ & $++/+++$ & - \\
\hline Occipital & - & + & $-/++$ & $-/+$ & $-/+$ & $-/+$ & $-1+$ \\
\hline Cingulate & $+/+++$ & +++ & ++ & $+/++$ & $-/+$ & +++ & - \\
\hline Insular & $+/+++$ & +++ & $+/+++$ & $+/++$ & $-/+$ & +++ & - \\
\hline \multicolumn{8}{|l|}{ Sub-cortical white matter } \\
\hline Frontal & N/A & N/A & $\mathrm{N} / \mathrm{A}$ & N/A & N/A & + & + \\
\hline Motor & N/A & N/A & $\mathrm{N} / \mathrm{A}$ & $\mathrm{N} / \mathrm{A}$ & N/A & + & $+/++$ \\
\hline Temporal & N/A & $\mathrm{N} / \mathrm{A}$ & $\mathrm{N} / \mathrm{A}$ & N/A & N/A & + & + \\
\hline Parietal & N/A & N/A & N/A & N/A & N/A & + & + \\
\hline Occipital & N/A & N/A & N/A & $\mathrm{N} / \mathrm{A}$ & N/A & + & $-/+$ \\
\hline Cingulate & $\mathrm{N} / \mathrm{A}$ & N/A & N/A & N/A & N/A & + & + \\
\hline Internal capsule & N/A & $\mathrm{N} / \mathrm{A}$ & $\mathrm{N} / \mathrm{A}$ & N/A & N/A & $+/++$ & + \\
\hline External capsule & N/A & N/A & N/A & N/A & N/A & ++ & + \\
\hline Amygdala & $-/+++$ & $+/++$ & $++/+++$ & $+/++$ & - & +++ & $-/+$ \\
\hline \multicolumn{8}{|l|}{ Hippocampus } \\
\hline Dentate fascia & - & $++/+++$ & + & + & - & $+/++$ & - \\
\hline CA4 & $-/++$ & $+/++$ & $+/++$ & + & $-/+$ & $++/+++$ & $-/+$ \\
\hline CA3 & $-/+++$ & $-/+$ & $+/++$ & $-/+++$ & - & $++/+++$ & - \\
\hline CA2 & $+/+++$ & - & $-/++$ & $-/+++$ & - & $++/+++$ & - \\
\hline CA1 & $-/++$ & $+/+++$ & $+/++$ & $+/++$ & + & $++/+++$ & - \\
\hline Subiculum & $-1+$ & $+/++$ & $+/++$ & $+/++$ & $-/+$ & $++/+++$ & - \\
\hline Entorhinal cortex & $-/++$ & +++ & $+/++$ & $+/++$ & $-/+$ & $++/+++$ & - \\
\hline Transentorhinal cortex & $+/++$ & $++/+++$ & $+/+++$ & $+/++$ & $-/+$ & $++/+++$ & - \\
\hline Caudate & + & $-/+$ & $+/+++$ & ++ & $-/+$ & $++/+++$ & - \\
\hline Putamen & $-/+$ & $-/++$ & $+/+++$ & $++/+++$ & $-/+$ & $++/+++$ & - \\
\hline Globus pallidus & $-1+$ & $-/++$ & $-1+$ & - & - & + & $-1+$ \\
\hline Thalamus & - & - & $-/+$ & $-/++$ & - & $-/++$ & $-1+$ \\
\hline Subthalamic nucleus & - & - & - & $-/+$ & - & $-/++$ & $-1+$ \\
\hline Red nucleus & - & - & - & - & - & + & + \\
\hline$\|^{\text {rd }}$ nerve nucleus & - & $-/+$ & $+/++$ & $+/+++$ & - & $+/+++$ & - \\
\hline Substantia nigra & +++ & - & $-/++$ & $-/+++$ & $-/+$ & $++/+++$ & $-/+$ \\
\hline Locus coeruleus & $++/+++$ & - & $-/++$ & $-/+++$ & - & $++/+++$ & - \\
\hline Pontine nuclei & - & - & - & + & $-/+$ & + & $-/+$ \\
\hline Pontine base white matter & N/A & $\mathrm{N} / \mathrm{A}$ & $\mathrm{N} / \mathrm{A}$ & N/A & N/A & $+/++$ & $+/++$ \\
\hline Dorsal motor nucleus of vagus & $+/+++$ & - & $-/+$ & $-/+++$ & $-/+$ & $++/+++$ & - \\
\hline Twelfth nerve nucleus & - & - & - & - & - & + & - \\
\hline Inferior olive & $-/+$ & - & - & $-/++$ & - & + & - \\
\hline Cerebellar hemisphere Purkinje cells & $+/++$ & - & - & - & - & - & $\mathrm{N} / \mathrm{A}$ \\
\hline
\end{tabular}


Table 2 Summary of neuropathological findings in three cases of G51D mutation (Continued)

\begin{tabular}{|c|c|c|c|c|c|c|c|}
\hline Cerebellar hemisphere white matter & N/A & $\mathrm{N} / \mathrm{A}$ & N/A & $\mathrm{N} / \mathrm{A}$ & N/A & $+/++$ & $+/++$ \\
\hline Dentate nucleus & - & - & - & - & - & - & - \\
\hline
\end{tabular}

The range of scores is provided

Oligodendroglial a-synuclein: cytoplasmic inclusions usually with similar morphology to glial cytoplasmic inclusions of MSA, less frequently resembling coiled bodies N/A not applicable, NFT Neurofibrillary tangle

nerve cell loss in cases one and two where the temporal, cingulate and insular cortices were most severely affected while cortical neuronal loss in case three was no more than mild in any region. In all cases the $\alpha-$ synuclein pathology was most severe in the superficial and deep cortical laminae (Fig. 2b). Balloon neurons, identified by $\alpha \mathrm{B}$-crystallin immunohistochemistry, were quite numerous predominantly in the deep layers of the neocortex, particularly in the frontal, temporal, cingulate and insular cortex (data not shown). Balloon neurons were immunoreactive for $\alpha$-synuclein and showed weak immunopositivity for p62 and ubiquitin, staining for tau was negative. No argyrophilic grains were identified in the hippocampus using immunohistochemical staining for p62 and tau. There was no $A \beta$ deposition in any of the cases. TDP-43 and tau pathology are described in detail below.

In comparison, the $\mathrm{H} 50 \mathrm{Q}$ and the SNCA duplication cases had less severe $\alpha$-synuclein pathology, with LB or LN morphology and a distribution typical of PD (Table 3, Fig. 2a). Furthermore, neither case was observed to have $\alpha$-synuclein inclusions resembling the annular, crescent, or NFT-like pathology of the G51D cases. Neuronal loss was severe in the $\mathrm{SN}$ and moderate in the locus

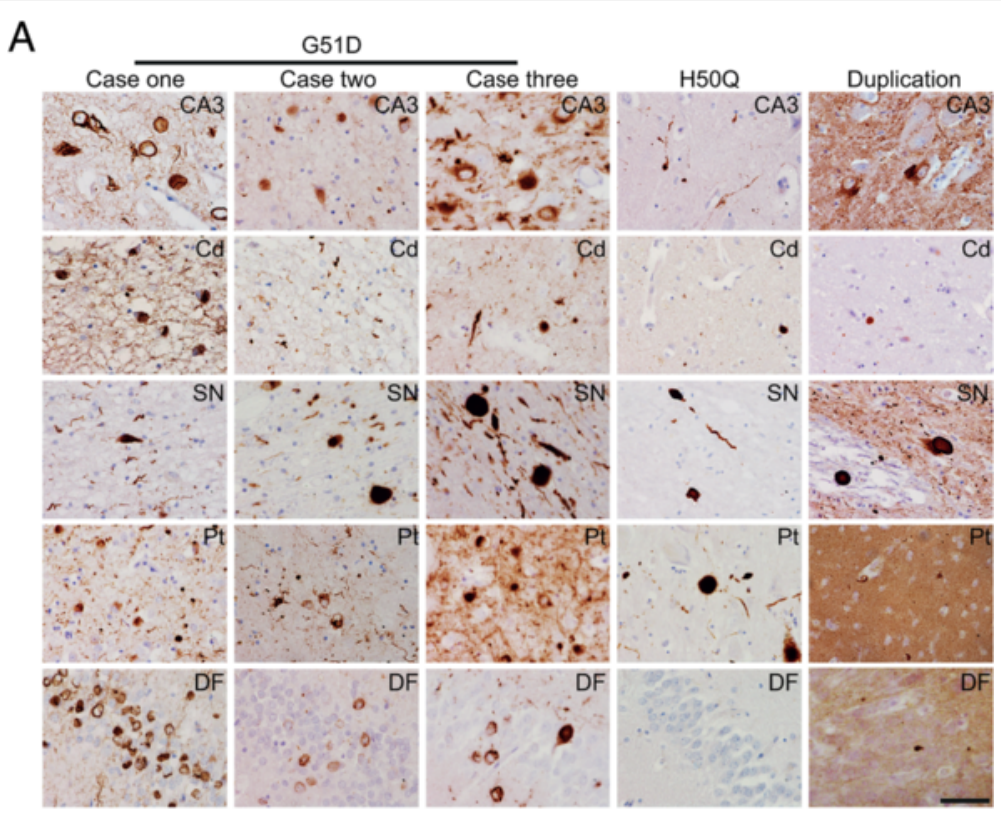

B

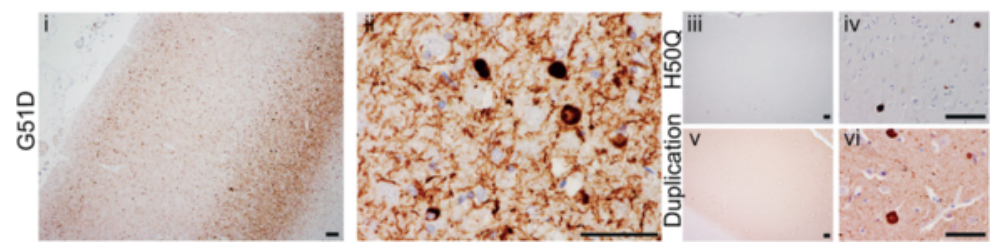

Fig. 2 a-Synuclein pathology. a Representative microscopy images of paraffin-embedded human brain tissue show abundant neuronal and neuritic a-synuclein pathology in three G51D cases compared to the H50Q mutation and SNCA duplication cases stained for a-synuclein protein. High magnification images from CA3, caudate $(C d)$, substantia nigra $(S N)$, putamen $(P t)$ and dentate fascia (DF). b Distinctive neocortical 'tramline' deposition of a-synuclein is only detected in G51D cases (i, ii), shown in representative low (i) and high (ii) magnification images of the entorhinal cortex, while in H50Q (iii, iv) and SNCA duplication $(v, v i)$ a-synuclein deposition was detected only in the deep cortical layers (iv, vi). Scale bars represent $50 \mu \mathrm{m}$ 
Table 3 Summary of neuropathological findings

\begin{tabular}{|c|c|c|c|c|c|}
\hline Pathology & Case 1 (G51D) & Case 2 (G51D) & Case 3 (G51D) & $\mathrm{H} 50 \mathrm{Q}$ & SNCA duplication \\
\hline Cortical neuronal loss & $\begin{array}{l}\text { Widespread. Severe in temporal } \\
\text { and insular, moderate in cingulate. }\end{array}$ & $\begin{array}{l}\text { Widespread. Severe in cingulate, } \\
\text { moderate in temporal and insular. }\end{array}$ & Widespread mild & None identified & None identified \\
\hline Hippocampal neuronal loss & CA2/3 predominant & CA2/3 predominant & CA2 mild & None identified & None identified \\
\hline Caudate neuronal loss & Mild & Mild & Mild & None identified & None Identified \\
\hline Brain stem neuronal loss & $\begin{array}{l}\text { Substantia nigra, locus coeruleus } \\
\text { and dorsal motor nucleus of vagus }\end{array}$ & $\begin{array}{l}\text { Substantia nigra, locus coeruleus } \\
\text { and dorsal motor nucleus of vagus }\end{array}$ & $\begin{array}{l}\text { Substantia nigra, locus } \\
\text { coeruleus and dorsal } \\
\text { motor nucleus of vagus }\end{array}$ & Substantia nigrab & $\begin{array}{l}\text { Substantia nigra, locus } \\
\text { coeruleus and dorsal motor } \\
\text { nucleus of vagus }\end{array}$ \\
\hline Neuronal a-synuclein pathology & $\begin{array}{l}\text { Annular, crescentic, globular, diffuse, } \\
\text { NFT-like. Widespread with severe } \\
\text { cortical involvement }\end{array}$ & $\begin{array}{l}\text { Annular, crescentic, globular, } \\
\text { diffuse, NFT-like. Widespread } \\
\text { with severe cortical involvement }\end{array}$ & $\begin{array}{l}\text { Annular, crescentic, globular, } \\
\text { diffuse, NFT-like. Widespread } \\
\text { with severe cortical involvement }\end{array}$ & PD type, Braak stage 6 & PD type, Braak stage 6 \\
\hline Glial a-synuclein pathology & GCI-like, rarely coiled body type & GCl-like, rarely coiled body type & GCl-like, rarely coiled body type & Absent & Sparse coiled-body type \\
\hline $\begin{array}{l}\text { Phosphorylated tau Braak and } \\
\text { Braak stage }\end{array}$ & $\|^{\mathrm{a}}$ & $\|^{\mathrm{a}}$ & $\|^{\mathrm{a}}$ & \|\|$^{a}$ & । \\
\hline$A \beta$ deposition & Absent & Absent & Absent & $\begin{array}{l}\text { Frequent diffuse } \\
\text { and sparse mature } \\
\text { cortical deposits }\end{array}$ & $\begin{array}{l}\text { Sparse diffuse neocortical } \\
\text { deposits }\end{array}$ \\
\hline TDP-43 pathology & Hippocampus, amygdala, striatum & $\begin{array}{l}\text { Hippocampus, amygdala, rare } \\
\text { in striatum }\end{array}$ & Absent & Absent & Absent \\
\hline
\end{tabular}

PD Parkinson's disease, NFT neurofibrillary tangle

$\mathrm{a}=$ dentate fascia also affected

$\mathrm{b}=$ locus coeruleus and dorsal motor nucleus of vagus not represented in available sections 
coeruleus and in both sites LBs and LNs were present. No neocortical neuronal loss was identified. Neocortical inclusions with features of cortical LBs were present in only moderate numbers and were most prominent in the deep cortical laminae in contrast to the pattern observed in the G51D cases (Fig. 2b). Lewy pathology corresponded to Braak stage 6 in both the SNCA duplication and the H50Q cases. GCI-like inclusions were not present in either case although the duplication case did have rare coiled body-like inclusions in the cerebellar and cerebral hemispheric subcortical white matter (Fig. 5b). There was no TDP-43 pathology in the hippocampus, amygdala or striatum in either case. Limited $A \beta$ deposition with sparse diffuse deposits in the temporal cortex and tau neurofibrillary tangle pathology corresponding to Braak and Braak stage I were observed in the SNCA duplication case. In the H50Q case numerous diffuse and sparse mature $A \beta$ cortical deposits were present, while neurofibrillary tangle pathology corresponded to Braak and Braak stage III and also involved the DF where there were sparse neurofibrillary tangles. Argyrophilic grains were not observed.

\section{Conformation and phosphorylation of a-synuclein}

We analysed the morphology and phosphorylation state of $\alpha$-synuclein within inclusions in order to predict the conformation of the protein. The 5G4 $\alpha$-synuclein antibody was used as it specifically detects high molecular weight $\alpha$-synuclein oligomers which are nitrated and have $\beta$-sheet conformation, with less binding to $\alpha$ synuclein fibrils and none to monomeric $\alpha$-synuclein $[14,15]$ (Fig. 3). We observed 5G4 immunoreactivity in all inclusion types in the G51D, H50Q and SNCA duplication cases.

We have previously shown that $\alpha$-synuclein inclusions in case one are phosphorylated at both the S129 and Y125 epitopes, which is of interest as phosphorylation at the S129 epitope is believed to promote-aggregation into fibrils while Y125 phosphorylation is suggested to result in oligomer formation [16-18]. On investigation of phosphorylation epitopes S129 and Y125 using specific antibodies, we observed that in both the H50Q and the $S N C A$ duplication cases only LB were positive for phosphorylated $\alpha$-synuclein, while LN were seldom immunoreactive indicating low levels of phosphorylated $\alpha$-synuclein. In contrast in the G51D cases all neuronal and neuritic inclusions were strongly labelled with the antibodies recognising $\alpha$-synuclein phosphorylated at both the S129 and Y125 epitopes (Fig. 4).

\section{Inclusions are ubiquitin and P62 positive}

Ubiquitin is a small molecule which can become covalently bound to proteins in an event called ubiquitination which is believed to signal for degradation of that

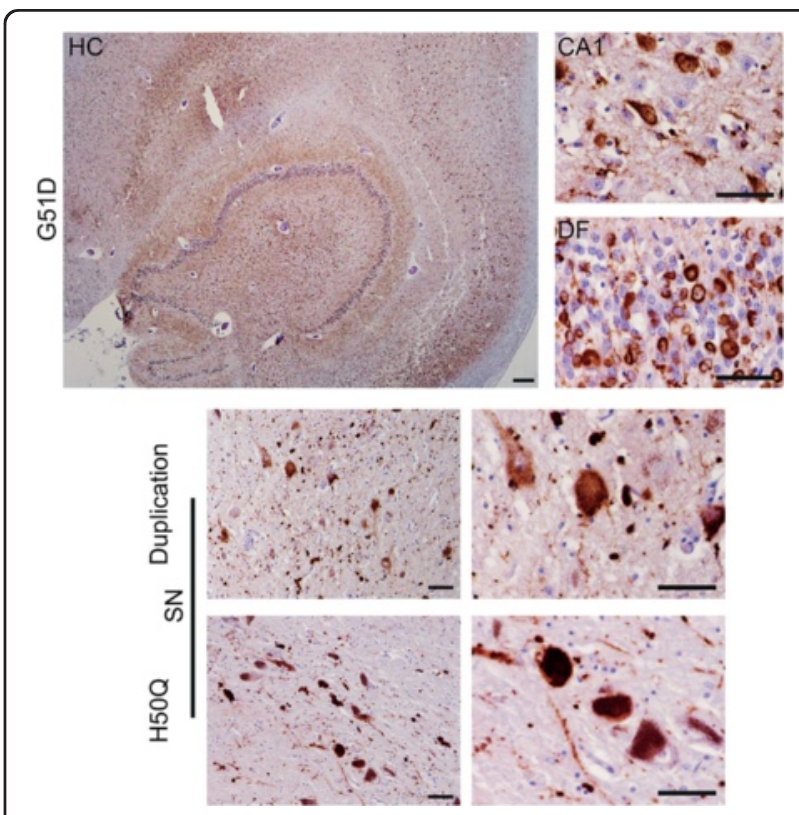

Fig. $35 \mathrm{G} 4$ a-synuclein. The $5 \mathrm{G} 4 \mathrm{a}$-synuclein antibody has high affinity for high molecular weight a-synuclein oligomers with lesser affinity for fibrils and low affinity for monomeric a-synuclein. Representative images show $5 \mathrm{G} 4$ positive a-synuclein accumulation in areas of severe inclusion burden in G51D (HC, hippocampus, CA1, cornu ammonis 1, DF, dentate fascia) SNCA duplication and H50Q (SN, substantia nigra). Scale bars represent $50 \mu \mathrm{m}$

protein via the ubiquitin-proteosomal system [19]. P62 recognises ubiquitinated proteins during autophagy and it has been shown that levels of p62 tend to inversely correlate with clearance of aggregated proteins via autophagy [20]. Double immunofluorescence microscopy for ubiquitin (Additional file 1A) or p62 (Additional file 1B) with $\alpha$-synuclein showed that neuronal and neuritic $\alpha$-synuclein inclusions in all cases were ubiquitinated. Co-localisation of p62 with $\alpha$-synuclein was observed in many neuronal inclusions including Lewy bodies but was less prominent in neuritic inclusions.

\section{GCI-like inclusions occur only in G51D not H50Q or SNCA duplication cases}

In all three G51D mutation cases $\alpha$-synuclein GCI-like inclusions were detected. These were confirmed as being within oligodendrocytes by double immunofluorescence with the oligodendrocyte marker Olig2 and were detected particularly in the sub-cortical white matter, cerebellar white matter and pons. GCI-like inclusions were observed to be of juxtanuclear conical, rod shaped or globular morphology (Fig. 5a).

The same double immunofluorescence technique did not reveal any oligodendroglial $\alpha$-synuclein inclusions in the H50Q case. Sparse, elongated $\alpha$-synuclein positive 


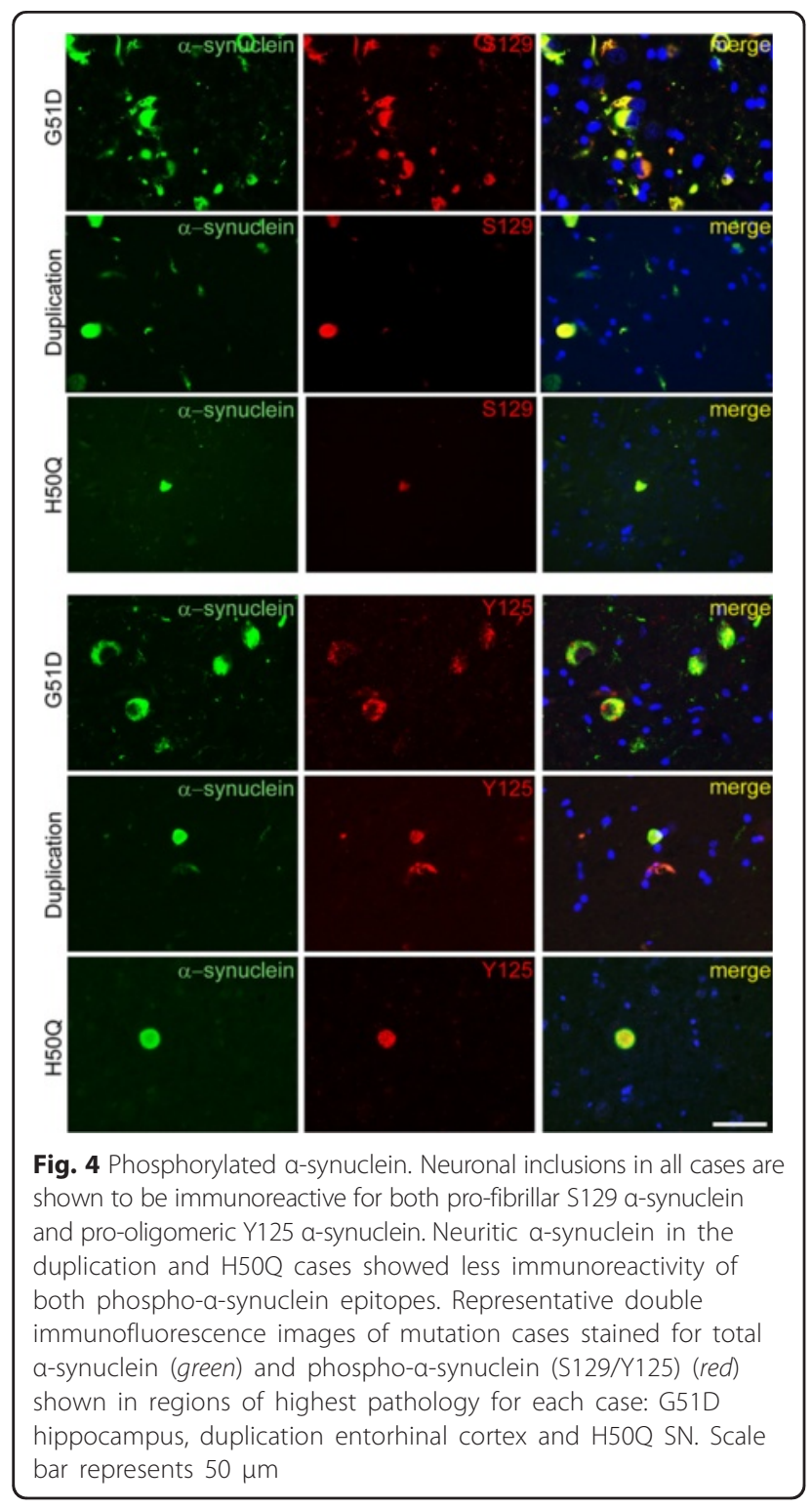

coiled body-like inclusions within oligodendrocytes of the cerebellar white matter were seen in the SNCA duplication case (Fig. 5b).

We observed rare instances in all G51D cases, in which $\alpha$-synuclein pathology co-localised with microglia as detected using the microglial marker Iba-1 (Fig. 5c, arrows and inset). Co-localisation was confirmed by confocal analysis. There was gliosis in affected regions of both the H50Q and the duplication case, but $\alpha$ synuclein co-localisation with microglia could not be identified. None of the cases were found to have $\alpha$ synuclein immunoreactive inclusions within astrocytes (data not shown).

Phospho-tau and a-synuclein co-localise in a subset of neuronal inclusions in G51D cases, and very rarely in the duplication case and the $\mathrm{H} 50 \mathrm{Q}$ case

Tau pathology was considered to correspond to Braak and Braak stage II in all G51D cases, although it was noted that there were also sparse to moderate numbers of neurofibrillary tangles in the granule cells of the DF. Argyrophilic grains were not found in any of the cases.

In our previous study we showed that a proportion of phospho-tau inclusions co-localised with a subset of $\alpha$ synuclein inclusions within neurons. Therefore, we were interested to determine whether this is a consistent finding in G51D cases two and three and to determine whether this might also be a feature of the H50Q and SNCA duplication cases, which had Braak and Braak stages III and I tau pathology, respectively. We used double immunofluorescence of phospho-tau (AT8) with $\alpha$-synuclein and in each case examined regions of the hippocampus and entorhinal cortex in which phosphotau pathology was most severe.

In keeping with our previous findings, a subset of the $\alpha$-synuclein inclusions was found to co-localise with tau inclusions in the hippocampus. Whether this subtle

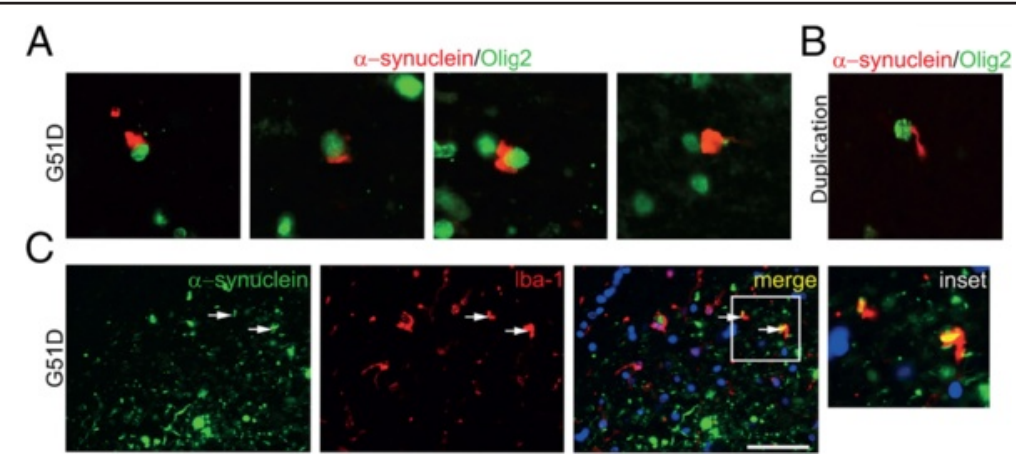

Fig. 5 Glial pathology. a The variable morphology of GCl-like inclusions in all three G51D cases is shown in representative images in which a-synuclein (red) is detected within oligodendrocytes (Olig2, green). b Rare coiled body-like inclusions of a-synuclein (red) are detected within oligodendrocytes (Olig2, green) within the cerebellar white matter of the duplication case. c On rare occasions a-synuclein (green) was confirmed to be present within microglia (Iba-1, red) in G51D cases (arrows) shown at high magnification (inset). Scale bar represents $50 \mu \mathrm{m}$ 
association is linked with the biology of the G51D mutant $\alpha$-synuclein is unclear. In contrast, in the H50Q case, in which tau pathology corresponding to Braak and Braak III with scarce hippocampal or entorhinal $\alpha$ synuclein inclusions, co-localisation of $\alpha$-synuclein with tau inclusions was limited to rare cells containing fine granular cytoplasmic structures (Fig. 6, arrows). In the duplication case co-localisation events, although robust, were very rare (Fig. 6) some partial co-localisation of $\alpha$ synuclein with rare neuritic tau was also detected.

\section{A subset of G51D a-synuclein inclusions is also immunoreactive for TDP-43}

TDP-43 positive intraneuronal cytoplasmic inclusions were a feature in the hippocampus, amygdala and striatum of G51D case one and case two (Fig. 7) but were absent from case three and could not therefore be considered a consistent feature of the disease (Table 3).

By use of double immunofluorescence for $\alpha$-synuclein with TDP-43 or phospho-TDP-43, we observed that a subset of TDP-43 inclusions in the CA regions, entorhinal cortex and DF of cases one and two co-localised with $\alpha$-synuclein inclusions. Representative images of colocalisation of TDP-43 and $\alpha$-synuclein inclusions are shown in Fig. 7a-f. All TDP-43 inclusions which colocalised with $\alpha$-synuclein inclusions in G51D cases were also immunoreactive for pTDP-43, as shown in the entorhinal cortex in Fig. 7, g-i. TDP-43 pathology was not detected in either the H50Q or the duplication case.

\section{Discussion}

We have established that consistent clinical and neuropathological features which resemble both those of PD and MSA characterise three G51D SNCA mutation cases. The three G51D SNCA mutation cases described in this study shared a stereotyped constellation of parkinsonian features with variable levodopa response, dementia, persistent visual hallucinations and autonomic dysfunction. All G51D cases had consistent neuropathological hallmarks which resembled both PD and MSA. These included widespread cortical and subcortical neuronal $\alpha$-synuclein inclusions together with small numbers of GCI-like inclusions in oligodendrocytes. The principal clinical phenotype of the duplication case bore some similarity to the G51D cases; parkinsonism, dementia and autonomic symptoms were all features. By comparison, the $\mathrm{H} 50 \mathrm{Q}$ case had a clinical phenotype consistent with idiopathic PD. Unlike the G51D cases, the neuropathology of the duplication and H50Q cases closely resembled idiopathic PD and GCI-like inclusions were not found in either case.

Predominant frontal release signs, executive dysfunction, perseveration, emotional lability and marked behavioural changes were observed in G51D cases two and three and in the SNCA duplication case. All three G51D cases had a good initial levodopa response, but the therapeutic efficacy was transient in cases two and three in which disease progression was rapid. Age of onset in G51D cases was variable, ranging from 19 (case one) to 69 (case two). There appears to be interfamilial
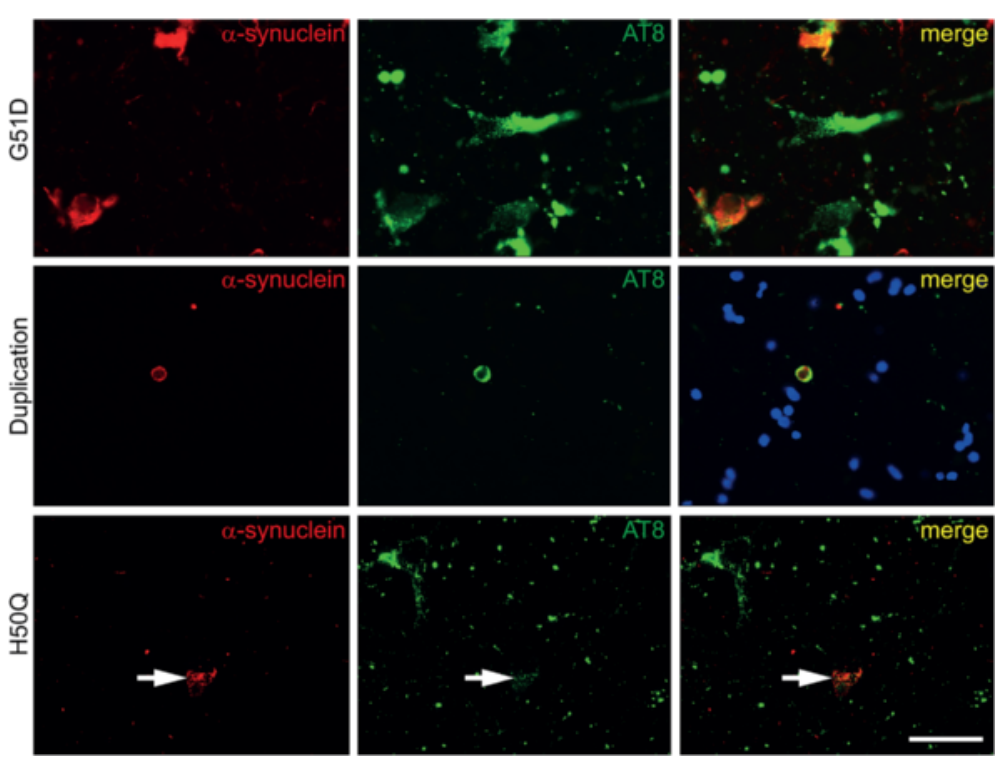

Fig. 6 Tau pathology. Double immunofluorescence images of phospho-tau (AT8, green) with a-synuclein (red) shows co-localisation in a subset of inclusions in G51D cases (shown in CA1) and very rarely in the duplication case (subiculum). Rare examples of sparse diffuse granular co-localisation was observed in the subiculum of the H50Q case (arrows). Scale bar represents $50 \mu \mathrm{m}$ 

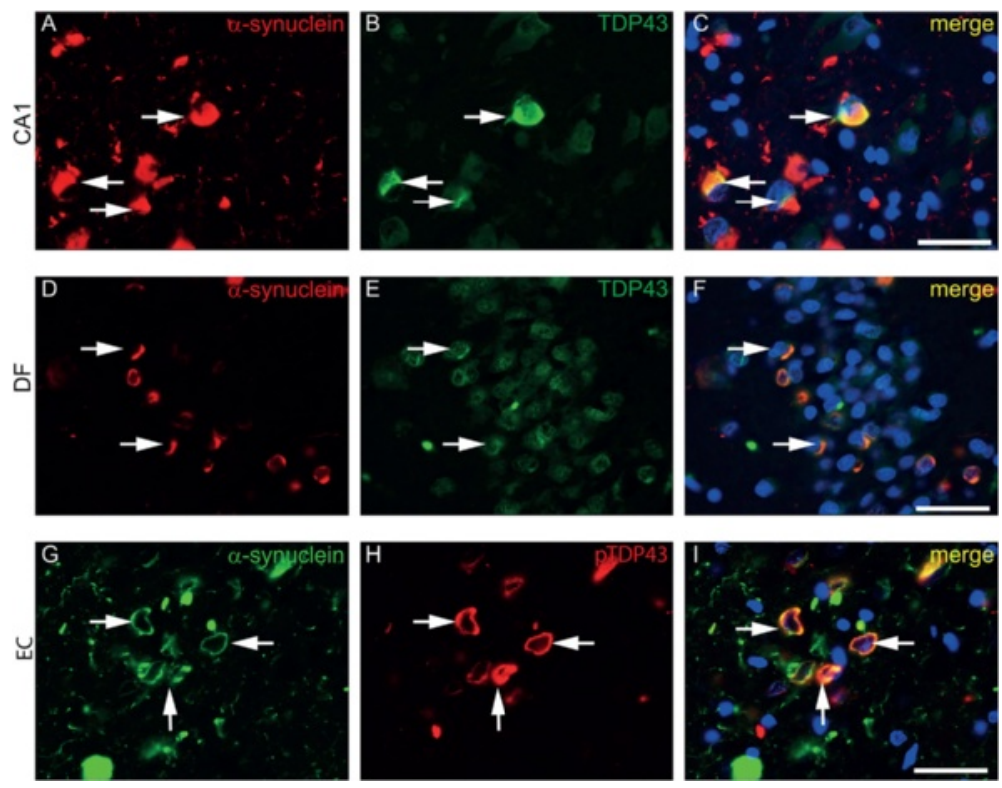

Fig. 7 TDP-43 pathology in G51D cases. Double immunofluorescence images show co-localisation of a moderate number of a-synuclein inclusions with TDP-43 (a-f) or pTDP-43 (g-i). Representative double immunofluorescence images of a-synuclein (red) with TDP-43 (green) indicate that TDP-43 inclusions in the CA and entorhinal cortex (EC) co-localise with a subset of a-synuclein inclusions, these events are more rare in the DF (arrows). Scale bar represents $50 \mu \mathrm{m}$

variability in the temporal course of disease progression. The second family (Fig. 1b) had a much more rapid deterioration and both cases died only 6 years after symptom onset. Both had an early onset of cognitive decline and loss of levodopa benefit. Significant motor fluctuation was only observed in the first family (Fig. 1a) in which the phenotype resembled that of a monogenetic parkinsonism such as parkin disease, with sustained levodopa response and longer disease duration spanning at least a decade [21]. In both families, depression and anxiety were early features that accompanied the onset of motor symptoms, followed by vivid dreams, intermittent disorientation, word-finding difficulties signifying memory impairment and paranoid delusions. Visual hallucinations were often triggered by a small increase in the dose of dopaminergic medications and would initially be amenable to the adjustment of antiparkinsonian medications or the introduction of a cholinesterase inhibitor. In more advanced stages of disease, visual hallucinations and behavioural changes became persistent and refractory, eventually dominating the clinical picture along with severe akinetic rigidity and bulbar dysfunction. Dysautonomia including symptomatic postural hypotension, urinary frequency and urge incontinence occurred after the onset of parkinsonism, but was a relatively early feature in G51D SNCA families when compared to idiopathic Parkinson's disease. In both families, the constellation of pathological reflexes, extensor plantar responses and autonomic dysfunction resembles the clinical phenotype of multiple system atrophy, specifically MSA with predominant parkinsonism (MSA-P), but the significant features of dementia with visual hallucinations are a useful pointer to set G51D SNCA mutation apart [22]. G51D mutation case two also had some features of a progressive supranuclear palsy phenotype, with unequivocal vertical supranuclear gaze palsy, blepharospasm and emotional lability.

Review of the clinical features reported in the literature in cases of SNCA duplication shows a phenotype of parkinsonism, frequently associated with dementia. Autonomic symptoms were observed in $50 \%$ of cases analysed [23]. Case-to-case variability has been reported in relation to age at onset, levodopa responsiveness and motor fluctuations [24, 25]. In our duplication case [12], the clinical syndrome was compatible with frontotemporal dementia with parkinsonism, prompting a tentative clinical diagnosis of FTDP-17 prior to genetic analysis (Fig. 1c). Cervical dystonia, blepharospasm and pyramidal signs were among the other atypical features observed in this case. In contrast, the H50Q SNCA mutation case resembled late-onset idiopathic Parkinson's disease with slow disease progression, sustained levodopa response and the absence of significant dysautonomia, pyramidal signs or dementia. Despite lack of known family history, this case may have a common ancestor with a further H50Q mutation case which has been described in a family with a history of PD [26], suggesting reduced penetrance rather than de novo mutation. The reported case had levodopa- 
responsive parkinsonism without pyramidal or cerebellar signs and, at time of publication, had most recently scored 23 of 30 in the Montreal cognitive assessment indicating mild cognitive impairment. More extensive characterisation of the clinical phenotype awaits the identification of more cases.

Detailed post-mortem examination of the brains of three G51D cases, one H50Q case and a SNCA duplication case was performed. The regional and cellular distribution of pathology was assessed in the G51D mutation cases with semi-quantitative assessment of the pathological changes. Despite some variability between cases in the regional severity of the pathology, cases two and three showed marked similarity with case one, the index case we previously reported [6]. All cases had widespread neuronal $\alpha$-synuclein pathology with marked neocortical involvement displaying the pattern of severely affected superficial and deep cortical laminae. In dementia with Lewy bodies (DLB) the deeper cortical laminae are affected first with involvement of superficial layers seen only with very advanced disease [27]. In contrast to DLB the G51D mutation cases display marked variability in the morphology of neuronal inclusions with many annular and crescentic inclusions in addition to those with appearances more typical of Lewy bodies. The striking involvement of the striatum with $\alpha$-synuclein neuronal inclusions and threads in case one was mirrored in case two although case three showed only moderate pathology. The neuropathological features of all G51D cases contrasted with those in the H50Q and SNCA duplication cases, both of which had an $\alpha$-synuclein inclusion distribution pattern of typical idiopathic PD consisting of LB and LN in brain stem, limbic and neocortical regions corresponding to Braak stage 6 disease [28]. In the SNCA duplication case rare $\alpha$-synuclein immunoreactive oligodendroglial coiled body-like inclusions were rarely noted as previously described in PD but no GCI-like inclusions were found in either the H50Q or SNCA duplication case.

Cell loss in the CA2-CA3 region of the hippocampus, which was prominent in the index G51D case, varied in severity between cases indicating that this pattern of cell loss is not a constant feature of the disease. TDP-43 pathology has been described in around $7 \%$ of PD cases and $19 \%$ of cases with PD dementia while it is rare in MSA [29, 30]. In keeping with these observations TDP43 positive inclusions were also found to be inconsistent between cases being a prominent feature in only two of the G51D cases and were absent in both the SNCA duplication and $\mathrm{H} 50 \mathrm{Q}$ mutation case. Sparse GCI-like oligodendroglial inclusions were observed in the white matter in all cases. This suggests that TDP-43 does not have a pathogenic role in these cases.

It is interesting that the neuropathological features that we initially described in association with G51D SNCA mutation [6] are similar to those subsequently described in a case with A53E mutation [7]. The A53E mutation case had similarly abundant $\alpha$-synuclein pathology of variable morphology in neurons and also displayed GCI-like oligodendroglial inclusions. The striatum was severely affected and they observed a similar 'tramline' like deposition of $\alpha$ synuclein pathology in the deep and superficial layers of the cortex. The authors did note differences, for example they observed only mild cell loss in the CA2-CA3 region. This was of interest as our G51D cases two and three showed milder neuronal loss in these regions and thus had greater similarity to the A53E case than to our initial case. In common with our observations in cases one and two Pasanen and colleagues [7] described TDP-43 inclusions in the DF, amygdala and striatum. In our previous publication we compared the neuropathological features of G51D case one with other SNCA mutations [6] (Table 2). The strongest similarities were observed between the G51D case and a reported SNCA triplication case as well as A53T mutation cases, which were also reported to show accumulation of GCIs, $\alpha$-synuclein pathology in the striatum and severe CA2/3 neuronal loss. This evidence supports the concept that different mutations of $\alpha$-synuclein can modify pathological changes and influence the pathways leading to neuronal or glial protein aggregation. Based on the proposed functions of $\alpha$-synuclein, several pathomechanisms have been suggested by which $\alpha$-synuclein may mediate or contribute to cell death including aberration of synaptic signalling [31, 32], mitochondrial dysfunction [33] and loss of chaperone function [34]. Both mutations and post translational modifications including phosphorylation, ubiquitination, nitration and glycosylation [35-38] have been shown to contribute to disease pathogenesis.

We explored the co-localisation of TDP-43 with $\alpha$ synuclein within inclusions showing that this occurred in a subset of inclusions and this was more common in the CA2-CA3 region than in the DF. Interestingly it was also in these neurons of the CA regions, DF and entorhinal cortex in which we observed co-localisation of $\alpha$ synuclein with phosphorylated tau in a subset of neurons in all three G51D cases. Although we were unable to investigate this in the current study, this suggests that $\alpha$ synuclein, tau and TDP-43 pathology could potentially all be present together in a proportion of these neurons. This feature in two of our three G51D cases resembles that of a case of corticobasal degeneration, which was reported to show partial co-localisation of $\alpha$-synuclein, TDP-43 and tau in inclusions supporting the concept of 'cross-seeding' of pathology [39]. The coexistence of tau with $\alpha$-synuclein in inclusions is not a new observation, tau has long been known to be present in LBs of both PD and Alzheimer's disease with amygdala LBs, especially in neurons which are particularly vulnerable to tau pathology [40]. Co-localisation of $\alpha$-synuclein and tau as 
hybrid oligomeric species may also occur in PD and DLB [41].

The phosphorylation of $\alpha$-synuclein has been reported, based on in vitro studies, to either promote (S129) fibrillisation/aggregation or prevent aggregation and promote oligomerisation (Y125) [16-18]. We have shown that $\alpha$ synuclein inclusions in G51D cases are frequently phosphorylated at both the S129 and Y125 epitopes. In contrast fewer $\alpha$-synuclein structures in the H50Q and duplication cases were found to be phosphorylated. This could indicate that the G51D mutation leads to a protein conformation which predisposes to phosphorylation. Furthermore, $\alpha$-synuclein is reported to be cleaved by cathepsin D at Y125 and phosphorylation of this epitope may prevent lysosomal degradation of $\alpha$-synuclein [42]. Thus the high degree of phosphorylation and abundance of aggregated $\alpha$-synuclein, detected using the 5G4 antibody, could suggest that pathological hyperphosphorylation leads to impaired clearance [43], which favours the development of the abundant cellular $\alpha$-synuclein inclusions characteristic of G51D mutation. We also showed co-localisation of ubiquitin and p62 with $\alpha$-synuclein in inclusions in all five cases. Both p62 and ubiquitin play important roles inthe proposed mechanisms of $\alpha$ synuclein proteolysis and our observations point to the necessity for future studies to investigate the role of impaired protein degradation in cases with $S N C A$ mutation.

The G51D and H50Q SNCA mutations are immediately adjacent in the putative protein loop that results in the hairpin formation of $\alpha$-synuclein protein [8]. $\alpha$ Synuclein has been proposed to form tetramers endogenously which resist disease-associated aggregation [44-46], although this proposed structure is a matter of on-going debate [47]. Disruption of the protein loop is believed to impair tetramer formation making mutant $\alpha$ synuclein monomers more susceptible to oligomerisation and aggregation [8]. It seems likely that each specific mutation of $\alpha$-synuclein affects the ability of the protein to form fibrillar aggregates to a different degree, resulting in distinct clinical [23] and neuropathological phenotype. This theory is supported by data presented here which shows the distinctly different phenotype of G51D cases compared to the H50Q case despite the fact that the sites of the mutations are immediately adjacent in the putative protein loop region.

The effect of SNCA point mutation on $\alpha$-synuclein aggregation has been a topic of discussion as a factor that may contribute to inclusion formation. In general the insight gained from investigations into in vitro disease models has been limited as they do not account for the contribution of factors such as dysfunction of clearance mechanisms [48] and neuroinflammation $[49,50]$ as in the disease state. The A53T and E46K mutant $\alpha$ synuclein proteins are reported to aggregate more rapidly [51-53] than the wild-type (WT) protein while the A30P has a more uncertain effect $[54,55]$. The G51D and H50Q mutations have also been reported to have variable effects on $\alpha$-synuclein aggregation. The G51D mutation has been reported to result in decreased aggregation [10,56] increased oligomer formation and significantly increased cell toxicity in the wake of stressors $\mathrm{H}_{2} \mathrm{O}_{2}$ and MPP+ treatment [57]. While the H50Q mutation has been reported to aggregate into fibrils more rapidly, it formed oligomers less readily than G51D or WT $\alpha$-synuclein and there was a trend towards increased cell toxicity in response to stressors in culture [57-61]. Neither mutant was shown to result in increased inclusion formation in cultured cells. The A53E mutation has also been reported to cause increased oligomer formation compared to WT protein [58]. The readiness with which the G51D and A53E mutant proteins form oligomers could prove to be relevant in understanding their associated pathology if the oligomeric species are the more toxic forms of the protein $[62,63]$.

The $\alpha$-synuclein 5G4 antibody $[14,15]$ was shown to specifically detect $\alpha$-synuclein oligomers in a high molecular weight, nitrated, $\beta$-sheet conformation and to have lesser affinity for fibrils and not to bind the disordered oligomers or monomers found in synaptic termini [15], We used the $5 \mathrm{G} 4$ antibody to demonstrate that the accumulation of $\alpha$ synuclein in a $\beta$-sheet oligomeric conformation is widespread in G51D cases. In the H50Q and duplication cases all neuritic or LB inclusions were immunoreactive for 5G4, indicating that, at the time of death, inclusions in G51D cases are no less aggregated by comparison despite the in vitro data suggesting slower aggregation properties conferred by this mutation. Furthermore, we did not detect severe or widespread accumulation of inclusions in the H50Q case compared with the spectrum of pathology in idiopathic PD. If H50Q $\alpha$-synuclein does aggregate faster than G51D $\alpha$-synuclein in vivo, the short fibrils which it is reported to form [57] may exist only transiently and be cleared by normal mechanisms. Studies in disease cases have shown that in some the neuropathology of the most rapidly aggregating mutant $\alpha$-synuclein protein, A53T, bears some similarity to that of G51D and A53E mutations [7], including CA2 cell loss, and GCI-like inclusions [64] for review [6]. The neuropathology of the H50Q case bears greater similarity to that of the more slowly aggregating A30P mutant [65], which gives rise to a pathological phenotype resembling sporadic PD [66]. Some cases of SNCA duplication have been reported, like G51D, to have GCI-like inclusions', tramline' like cortical deposition of $\alpha$-synuclein pathology in the deep and superficial layers of the cortex and CA2-CA3 cell loss [67-69]. Although neuronal loss and $\alpha$-synuclein inclusions were more abundant and widespread in our duplication case than the H50Q case (Table 3), the pathology did not reach the severity of the 
G51D cases and resembled sporadic PD. Although only a single duplication case was available for this study, our findings indicate that increased $\alpha$-synuclein expression is not the sole factor which determines the abundance of $\alpha$ synuclein inclusions and neuronal loss. Altered conformation of the protein due to mutation may impede protein clearance mechanisms thus predisposing to a high load of $\alpha$-synuclein containing intracellular inclusions.

\section{Conclusions}

Here we have described the spectrum of clinical and neuropathological characteristics of a small series of G51D SNCA mutation cases providing information to facilitate the recognition of this clinicopathological entity. Our detailed analysis confirms that clinical features including variable levodopa response, dementia, persistent visual hallucinations and autonomic dysfunction were consistent in all three cases. The neuropathological features of all three G51D cases share characteristics of both PD and MSA these included widespread cortical and subcortical neuronal $\alpha$-synuclein inclusions together with small numbers of GCI-like inclusions in oligodendrocytes. We have shed light on the differential effects of SNCA mutations on neuropathology from which we have gained insight into the biology of pathological $\alpha$ synuclein. It is vital that we further our understanding of the biology of $\alpha$-synuclein in disease in order to identify potential pathways and mechanisms which can be targeted for therapeutic intervention.

\section{Methods}

\section{Clinical data}

Medical records, including notes from the general practitioners, letters from hospital specialists and in-patient notes, were retrospectively reviewed by a neurologist with a specialist interest in movement disorders (HL). Clinical symptoms and signs not documented in the notes were considered as absent. Where there was discrepancy in the clinical features described, the neurologists' record was used.

Consent for research was obtained for all cases included in the study.

\section{Brain tissue}

Three cases with G51D and one case with the H50Q SNCA mutation were donated to the Queen Square Brain Bank for Neurological Disorders, UCL Institute of Neurology. The donation protocols had Research Ethics Committee approval and the tissue was stored for research under a license issued by the Human Tissue Authority (No. 12198). Following fixation in $10 \%$ buffered formalin, the right half of the brain was sliced in the coronal plane, examined and blocks were selected for paraffin wax embedding and histology. The SNCA duplication case was donated to the Department of Neuropathology, North Bristol NHS Trust, Bristol with consent for research.

Paraffin-embedded sections $(8 \mu \mathrm{m})$ were stained with haematoxylin and eosin (H\&E) and Luxol fast blue/cresyl violet. Immunohistochemistry was performed as previously described [70] using primary antibodies (Additional file 2). Double immunofluorescence was detected using isotype specific anti-rabbit IgG or anti-mouse IgG secondary antibodies conjugated with either Alexa 488 or 568 fluorochromes (1:400) (Life technologies, Paisley, UK) followed by quenching or autofluorescence with $0.1 \%$ Sudan Black/70 \% ethanol (Sigma-Aldrich, Dorset, UK) solution for $10 \mathrm{~min}$ and mounting under glass coverslips using VECTAshield mounting media with 4,6-diamidino2-phenylindole (DAPI) nuclear stain (Vector laboratories, Peterborough, UK). Images were visualised using a confocal fluorescence microscopy (Leica DM5500 B).

\section{Genetics}

Genetic analysis of G51D SNCA mutation cases two and three was performed using Sanger sequencing of the SNCA gene as previously described [6]. The H50Q case was described by Proukakis et al. [5] and the SNCA duplication case was identified using multiplex ligation dependent probe amplification (MLPA) and DNA array analysis, Kara et al. [12].

\section{Additional files}

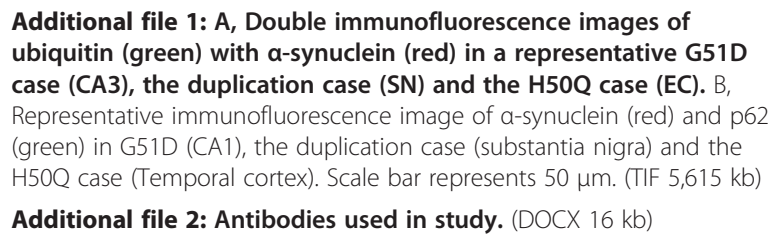

\section{Abbreviations}

PD: Parkinson's disease; MSA: Multiple system atrophy; GCls: Glial cytoplasmic inclusions; DLB: Dementia with Lewy bodies; LB: Lewy bodies; LN: Lewy neurites; CA: Cornu ammonis; SPECT: Single-photon emission computerised tomography; FTDP-17: Frontotemporal dementia with parkinsonism-17; MMSE: Mini mental state examination; Cd: Caudate; SN: Substantia nigra; Pt: Putamen; DF: Dentate fascia; MSA-P: MSA with predominant parkinsonism; HC: Hippocampus; EC: Entorhinal cortex.

\section{Competing interests}

The authors declare that they have no competing interests.

\section{Authors' contributions}

APK carried out immunohistochemical and immunofluorescence staining, microscopy and analysis and drafted the manuscript. HL analysed medical records of all cases and contributed to drafting the manuscript. YTA and S Love contributed to data acquisition and study design. EK and S Lubbe performed genetic analysis of the cases. CP, PAL and AHS contributed intellectual input and contributed to data analysis. PL, HRM, HCR, AJL, and NQ provided clinical data, intellectual input and arranged tissue acquisition. $\mathrm{HH}$ and $\mathrm{J}$ Hardy participated in the design of the study, genetic analysis and data interpretation. TR and JHolton conceived the study and participated in its design, supervised the study 
and helped to draft the manuscript. All authors reviewed and approved the final manuscript.

\section{Acknowledgments}

JLH and AJL are supported by Parkinson's UK (PUK), the Multiple System Atrophy (MSA) Trust and the Progressive Supranuclear Palsy (Europe) Association. TR and $\mathrm{JH}$ are supported by Cortico Basal Degeneration (CBD) Solutions. JLH is supported by Alzheimer's Research UK, the Michael J Fox Foundation. Queen Square Brain Bank is supported by Reta Lila Weston Insitute for Neurological Studies and the Medical Research Council UK. APK is supported by the MSA Trust. HL is supported by a CBD Solutions research grant and employed by Reta Lila Weston Institute of Neurological Studies. YTA is supported by the Government of Kuwait. HRM and SL are funded by PUK (Grants 8047 and J1101) and the Medical Research Council (MRC) UK (G0700943, G1100643). PAL is a PUK research fellow (F1002) and is supported by a New Investigator Research Grant from the MRC UK (MR/L010933/1). HH is supported by the MRC, the Dystonia Medical Research Foundation (DMRF) and the Parkinson's disease foundation. AHS is supported by PUK and is a National Institute for Health research (NIHR) senior investigator. The research was in part funded by the NIHR Biomedical Research Unit in Dementia based at University College London Hospitals (UCLH), University College London (UCL). The views expressed are those of the author(s) and not necessarily those of the NHS, the NIHR or the Department of Health. This work was supported in part by the Wellcome Trust/MRC Joint Call in Neurodegeneration award (WT089698) to the UK Parkinson's Disease Consortium (UKPDC) whose members are from the UCL Institute of Neurology, the University of Sheffield and the MRC protein Phosphorylation Unit at the University of Dundee. This research was supported by the National Institute for Health Research University College London Hospitals Biomedical Research Centre.

\section{Author details}

${ }^{1}$ Department of Molecular Neuroscience, Queen Square Brain Bank, UCL Institute of Neurology, Queen Square, WC1N 3BG London, UK. ²Department of Molecular Neuroscience and Reta Lila Weston Institute of Neurological Studies, UCL Institute of Neurology, London, UK. ${ }^{3}$ Sobell Department of Motor Neuroscience and Movement Disorders, Unit of Functional Neurosurgery, UCL Institute of Neurology, UCL, London, UK. ${ }^{4}$ School of Pharmacy, University of Reading, Whiteknights, Reading, UK. ${ }^{5}$ Department of Clinical Neuroscience, UCL Institute of Neurology, London, UK. ${ }^{6}$ Academic Geriatric Medicine, University of Southampton, Southampton, UK. ${ }^{7}$ National Hospital for Neurology and Neurosurgery, Queen Square, London, UK. ${ }^{8}$ Clinical Neurosciences, University of Bristol, Bristol, UK. ${ }^{9}$ Alzheimer's Disease Research Centre, Harvard medical school \& Massachusetts General Hospital, 114 16th Street, Charlestown, MA 02129, USA.

\section{Received: 22 May 2015 Accepted: 13 August 2015 Published online: 27 August 2015}

\section{References}

1. Lashuel HA, Overk CR, Oueslati A, Masliah E. The many faces of [alpha]-synuclein: from structure and toxicity to therapeutic target. Nat Rev Neurosci. 2013;14(1):38-48.

2. Polymeropoulos M, Lavedan C, Leroy E, Ide S, Dehejia A, Dutra A, et al. Mutation in the alpha-synuclein gene identified in families with Parkinson's disease. Science. 1997;276:2045-7.

3. Zarranz JJ, Alegre J, Gómez-Esteban JC, Lezcano E, Ros R, Ampuero I, et al, The new mutation, E46K, of a-synuclein causes parkinson and Lewy body dementia. Ann Neurol. 2004;55(2):164-73.

4. Kruger R, Kuhn W, Muller T, Woitalla D, Graeber M, Kosel S, et al. Ala30Pro mutation in the gene encoding alpha-synuclein in Parkinson's disease. Nat Genet. 1998;18:106-8.

5. Proukakis C, Dudzik CG, Breier T, MacKay DS, Cooper JM, Millhauser GL, et al. A novel alpha-synuclein missense mutation in Parkinson's disease. Neurology. 2012;80(11):1062-4.

6. Kiely AP, Asi Y, Kara E, Limousin P, Ling H, Lewis P, et al. a-Synucleinopathy associated with G51D SNCA mutation: a link between Parkinson's disease and multiple system atrophy? Acta Neuropathol. 2013;125(5):753-69.

7. Pasanen P, Myllykangas L, Siitonen M, Raunio A, Kaakkola S, Lyytinen J, et al. A novel a-synuclein mutation A53E associated with atypical multiple system atrophy and Parkinson's disease-type pathology. Neurobiol Aging. 2014.
8. Kara E, Lewis PA, Ling H, Proukakis C, Houlden H, Hardy J. a-Synuclein mutations cluster around a putative protein loop. Neurosci Lett. 2013;546:67-70.

9. Collaboration TM-SAR. Mutations in COQ2 in familial and sporadic multiple-system atrophy. N Engl J Med. 2013;369:233-44.

10. Lesage S, Anheim M, Letournel F, Bousset L, Honoré A, Rozas N, et al. G51D a-synuclein mutation causes a novel Parkinsonian-pyramidal syndrome. Ann Neurol. 2013;73(4):459-71.

11. Tokutake T, Ishikawa A, Yoshimura N, Miyashita A, Kuwano R, Nishizawa M, et al. Clinical and neuroimaging features of patient with early-onset Parkinson's disease with dementia carrying SNCA p.G51D mutation. Parkinsonism Relat Disord. 2014;20(2):262-4.

12. Kara E, Kiely AP, Proukakis C, Giffin N, Love S, Hehir J, et al. A 6.4 mb duplication of the a-synuclein locus causing frontotemporal dementia and parkinsonism: phenotype-genotype correlations. JAMA Neurol. 2014;71(9):1162-71.

13. Seidel K, Mahlke J, Siswanto S, Krüger R, Heinsen H, Auburger G, et al. The brainstem pathologies of Parkinson's disease and dementia with lewy bodies. Brain Pathol. 2014;25(2):121-35.

14. Kovacs G, Wagner U, Dumont B, Pikkarainen M, Osman A, Streichenberger $\mathrm{N}$, et al. An antibody with high reactivity for disease-associated a-synuclein reveals extensive brain pathology. Acta Neuropathol. 2012;124(1):37-50

15. Kovacs GG, Breydo L, Green R, Kis V, Puska G, Lőrincz P, et al. Intracellular processing of disease-associated a-synuclein in the human brain suggests prion-like cell-to-cell spread. Neurobiol Dis. 2014

16. Chen L, Periquet M, Wang X, Negro A, McLean PJ, Hyman BT, et al. Tyrosine and serine phosphorylation of a-synuclein have opposing effects on neurotoxicity and soluble oligomer formation. J Clin Invest. 2009;119(11):3257-65.

17. Hejjaoui M, Butterfield S, Fauvet B, Vercruysse F, Cui J, Dikiy I, et al. Elucidating the role of C-terminal post-translational modifications using protein semisynthesis strategies: a-synuclein phosphorylation at tyrosine 125. J Am Chem Soc. 2012;134(11):5196-210.

18. Negro A, Brunati AM, Donella-Deana A, Massimino ML, Pinna LA. Multiple phosphorylation of a-synuclein by protein tyrosine kinase Syk prevents eosin-induced aggregation. FASEB J. 2002;16(2):210-2.

19. Tai H-C, Schuman EM. Ubiquitin, the proteasome and protein degradation in neuronal function and dysfunction. Nat Rev Neurosci. 2008;9(11):826-38.

20. Komatsu M, Waguri S, Koike M, Sou Y-S, Ueno T, Hara T, et al. Homeostatic levels of p62 control cytoplasmic inclusion body formation in autophagy-deficient mice. Cell. 2007;131(6):1149-63.

21. Doherty KM, Silveira-Moriyama L, Parkkinen L, Healy DG, Farrell M, Mencacci $\mathrm{NE}$, et al. Parkin disease: a clinicopathologic entity? JAMA Neurol. 2013;70(5):571-9.

22. Asi YT, Ling H, Ahmed Z, Lees AJ, Revesz T, Holton JL. Neuropathological features of multiple system atrophy with cognitive impairment. Mov Disord. 2014;29(7):884-8.

23. Kasten $\mathrm{M}$, Klein $\mathrm{C}$. The many faces of alpha-synuclein mutations. Mov Disord. 2013;28(6):697-701. doi:10.1002/mds.25499. Epub 2013 May 14.

24. Fuchs J, Nilsson C, Kachergus J, Munz M, Larsson E-M, Schüle B, et al. Phenotypic variation in a large Swedish pedigree due to SNCA duplication and triplication. Neurology. 2007;68(12):916-22.

25. Nishioka K, Ross OA, Ishii K, Kachergus JM, Ishiwata K, Kitagawa M, et al. Expanding the clinical phenotype of SNCA duplication carriers. Mov Disord. 2009;24(12):1811-9.

26. Appel-Cresswell S, Vilarino-Guell C, Encarnacion M, Sherman H, Yu I, Shah B, et al. Alpha-synuclein p.H50Q, a novel pathogenic mutation for Parkinson's disease. Mov Disord. 2013;28(6):811-3.

27. Marui W, Iseki E, Nakai T, Miura S, Kato M, Uéda K, et al. Progression and staging of Lewy pathology in brains from patients with dementia with Lewy bodies. J Neurol Sci. 2002;195(2):153-9.

28. Braak H, Del Tredici K, Rub U, de Vos R, Jansen Steur E, Braak E. Staging of brain pathology related to sporadic Parkinson's disease. Neurobiol Aging. 2003;24:197-211.

29. Nakashima-Yasuda H, Uryu K, Robinson J, Xie S, Hurtig H, Duda J, et al. Co-morbidity of TDP-43 proteinopathy in Lewy body related diseases. Acta Neuropathol. 2007;114(3):221-9.

30. Geser F, Malunda JA, Hurtig HI, Duda JE, Wenning GK, Gilman S, et al. TDP-43 pathology occurs infrequently in multiple system atrophy. Neuropathol Appl Neurobiol. 2011;37(4):358-65. Epub 2010/10/15. 
31. Abeliovich A, Schmitz Y, Fariñas I, Choi-Lundberg D, Ho W-H, Castillo PE, et al. Mice lacking a-synuclein display functional deficits in the nigrostriatal dopamine system. Neuron. 2000;25(1):239-52.

32. Rohan de Silva HA, Khan NL, Wood NW. The genetics of Parkinson's disease. Curr Opin Genet Dev. 2000;10(3):292-8.

33. Nakamura K, Nemani VM, Azarbal F, Skibinski G, Levy JM, Egami K, et al. Direct membrane association drives mitochondrial fission by the Parkinson disease-associated protein a-synuclein. J Biol Chem. 2011;286(23):20710-26.

34. Chandra S, Gallardo G, Fernández-Chacón R, Schlüter OM, Südhof TC. a-synuclein cooperates with CSPa in preventing neurodegeneration. Cell. 2005;123(3):383-96.

35. Anderson J, Walker D, Goldstein J, de Laat R, Banducci K, Caccavello R, et al. Phosphorylation of Ser-129 is the dominant pathological modification of alpha-synuclein in familial and sporadic Lewy body disease. J Biol Chem. 2006;281:29739-52.

36. Giasson BI, Duda JE, Murray IVJ, Chen Q, Souza JM, Hurtig HI, et al. Oxidative damage linked to neurodegeneration by selective a-synuclein nitration in synucleinopathy lesions. Science. 2000;290(5493):985-9.

37. Tofaris GK, Razzaq A, Ghetti B, Lilley KS, Spillantini MG. Ubiquitination of a-synuclein in lewy bodies is a pathological event Not associated with impairment of proteasome function. J Biol Chem. 2003;278(45):44405-11.

38. Guerrero E, Vasudevaraju P, Hegde M, Britton GB, Rao KS. Recent advances in a-synuclein functions, advanced glycation, and toxicity: implications for Parkinson's disease. Mol Neurobiol. 2013;47(2):525-36.

39. Yamashita S, Sakashita N, Yamashita T, Tawara N, Tasaki M, Kawakami K, et al. Concomitant accumulation of a-synuclein and TDP-43 in a patient with corticobasal degeneration. J Neurol. 2014;1-9.

40. Ishizawa T, Mattila P, Davies P, Wang D, Dickson DW. Colocalization of Tau and alpha-synuclein epitopes in lewy bodies. J Neuropathol Exp Neurol. 2003;62(4):389-97.

41. Sengupta U, Guerrero-Muñoz MJ, Castillo-Carranza DL, Lasagna-Reeves CA, Gerson JE, Paulucci-Holthauzen AA, et al. Pathological interface between oligomeric alpha-synuclein and Tau in synucleinopathies. Biol Psychiatry. 2015.

42. Hossain S, Alim A, Takeda K, Kaji H, Shinoda T, Ueda K. Limited proteolysis of NACP/alpha-synuclein. J Alzheimers Dis. 2001;3:577-84

43. Tenreiro S, Reimão-Pinto MM, Antas P, Rino J, Wawrzycka D, Macedo D, et al. Phosphorylation modulates clearance of alpha-synuclein inclusions in a yeast model of Parkinson's disease. PLoS Genet. 2014;10(5), e1004302.

44. Selkoe D, Dettmer U, Luth E, Kim N, Newman A, Bartels T. Defining the native state of a-synuclein. Neurodegener Dis. 2014;13(2-3):114-7.

45. Bartels T, Choi JG, Selkoe DJ. alpha-Synuclein occurs physiologically as a helically folded tetramer that resists aggregation. Nature. 2011;477(7362):107-10. Epub 2011/08/16.

46. Dettmer U, Newman AJ, Luth ES, Bartels T, Selkoe D. In vivo cross-linking reveals principally oligomeric forms of a-synuclein and $\beta$-synuclein in neurons and Non-neural cells. J Biol Chem. 2013;288(9):6371-85.

47. Burre J, Vivona S, Diao J, Sharma M, Brunger AT, Sudhof TC. Properties of native brain [agr]-synuclein. Nature. 2013;498(7453):E4-6.

48. Atkin $\mathrm{G}$, Paulson H. Ubiquitin pathways in neurodegenerative disease. Front Mol Neurosci. 2014;7:63. doi:10.3389/fnmol.2014.00063. eCollection 2014.

49. Gao HM, Zhang F, Zhou H, Kam W, Wilson B, Hong JS. Neuroinflammation and alpha-synuclein dysfunction potentiate each other, driving chronic progression of neurodegeneration in a mouse model of Parkinson's disease. Environ Health Perspect. 2011;119(6):807-14. Epub 2011/01/20.

50. Brundin P, Li J-Y, Holton JL, Lindvall O, Revesz T. Research in motion: the enigma of Parkinson's disease pathology spread. Nat Rev Neurosci. 2008;9(10):741-5.

51. Greenbaum EA, Graves CL, Mishizen-Eberz AJ, Lupoli MA, Lynch DR, Englander SW, et al. The E46K mutation in a-synuclein increases amyloid fibril formation. J Biol Chem. 2005;280(9):7800-7.

52. Choi W, Zibaee S, Jakes R, Serpell LC, Davletov B, Anthony Crowther R, et al. Mutation E46K increases phospholipid binding and assembly into filaments of human a-synuclein. FEBS Lett. 2004;576(3):363-8

53. Conway KA, Harper JD, Lansbury PT. Accelerated in vitro fibril formation by a mutant alpha-synuclein linked to early-onset Parkinson disease. Nat Med. 1998:4(11):1318-20. Epub 1998/11/11.

54. Conway KA, Lee S-J, Rochet J-C, Ding TT, Williamson RE, Lansbury PT. Acceleration of oligomerization, not fibrillization, is a shared property of both a-synuclein mutations linked to early-onset Parkinson's disease: implications for pathogenesis and therapy. Proc Natl Acad Sci. 2000;97(2):571-6.

55. Narhi L, Wood SJ, Steavenson S, Jiang Y, Wu GM, Anafi D, et al. Both familial Parkinson's disease mutations accelerate a-synuclein aggregation. J Biol Chem. 1999;274(14):9843-6

56. Fares M-B, Bouziad NA, Dikiy I, Mbefo MK, Jovičić A, Kiely A, et al. The novel Parkinson's disease linked mutation G51D attenuates in vitro aggregation and membrane binding of a-synuclein, and enhances its secretion and nuclear localization in cells. Hum Mol Genet. 2014;23(17):4491-509.

57. Rutherford NJ, Moore BD, Golde TE, Giasson BI. Divergent effects of the H50Q and G51D SNCA mutations on the aggregation of a-synuclein. J Neurochem. 2014;131(6):859-67.

58. Ghosh D, Sahay S, Ranjan P, Salot S, Mohite GM, Singh PK, et al. The newly discovered Parkinson's disease associated Finnish mutation (A53E) attenuates a-synuclein aggregation and membrane binding. Biochemistry (Mosc). 2014;53(41):6419-21.

59. Chi Y-C, Armstrong GS, Jones DNM, Eisenmesser EZ, Liu C-W. Residue histidine 50 plays a Key role in protecting a-synuclein from aggregation at physiological pH. J Biol Chem. 2014;289(22):15474-81.

60. Khalaf O, Fauvet B, Oueslati A, Dikiy I, Mahul-Mellier A-L, Ruggeri FS, et al. The $\mathrm{H} 50 \mathrm{Q}$ mutation enhances a-synuclein aggregation, secretion, and toxicity. J Biol Chem. 2014;289(32):21856-76.

61. Porcari R, Proukakis C, Waudby CA, Bolognesi B, Mangione PP, Paton JFS, et al. The H50Q mutation induces a 10-fold decrease in the solubility of a-synuclein. J Biol Chem. 2015;290(4):2395-404

62. Winner B, Jappelli R, Maji SK, Desplats PA, Boyer L, Aigner S, et al. In vivo demonstration that a-synuclein oligomers are toxic. Proc Natl Acad Sci. 2011;108(10):4194-9.

63. Kalia LV, Kalia SK, McLean PJ, Lozano AM, Lang AE. a-Synuclein oligomers and clinical implications for Parkinson disease. Ann Neurol. 2013;73(2):155-69.

64. Markopoulou K, Dickson D, McComb R, Wszolek Z, Katechalidou L, Avery L, et al. Clinical, neuropathological and genotypic variability in SNCA A53T familial Parkinson's disease. Acta Neuropathol. 2008;116(1):25-35.

65. Lemkau LR, Comellas G, Kloepper KD, Woods WS, George JM, Rienstra CM Mutant protein A30P a-synuclein adopts wild-type fibril structure, despite slower fibrillation kinetics. J Biol Chem. 2012;287(14):11526-32.

66. Seidel K, Schöls L, Nuber S, Petrasch-Parwez E, Gierga K, Wszolek Z, et al. First appraisal of brain pathology owing to A30P mutant alpha-synuclein. Ann Neurol. 2010;67(5):684-9.

67. Obi T, Nishioka K, Ross OA, Terada T, Yamazaki K, Sugiura A, et al. Clincopathologic study of a SNCA gene duplication patient with Parkinson disease and dementia. Neurology. 2008;70(3):238-41.

68. Ikeuchi T, Kakita A, Shiga A, Kasuga K, Kaneko H, Tan CF, et al. Patients homozygous and heterozygous for snca duplication in a family with parkinsonism and dementia. Arch Neurol. 2008;65(4):514-9.

69. Gwinn-Hardy K, Mehta ND, Farrer M, Maraganore D, Muenter M, Yen SH, et al. Distinctive neuropathology revealed by alpha-synuclein antibodies in hereditary parkinsonism and dementia linked to chromosome $4 p$. Acta Neuropathol. 2000;99(6):663-72. Epub 2000/06/27.

70. Ozawa T, Paviour D, Quinn NP, Josephs KA, Sangha H, Kilford L, et al. The spectrum of pathological involvement of the striatonigral and olivopontocerebellar systems in multiple system atrophy: clinicopathological correlations. Brain. 2004;127(12):2657-71.

\section{Submit your next manuscript to BioMed Central and take full advantage of:}

- Convenient online submission

- Thorough peer review

- No space constraints or color figure charges

- Immediate publication on acceptance

- Inclusion in PubMed, CAS, Scopus and Google Scholar

- Research which is freely available for redistribution 